\title{
STUDYING STABILITY OF THE LIBERATION POINTS OFBINARY ASTEROIDES
}

M.N.Ismail ${ }^{1}$, A.Bakry ${ }^{1}$, Kh.I.Khalii ${ }^{2}$, A. Hafez ${ }^{1}$

${ }^{1}$ Astronomy and Metrology Department Faculty of Science, Al-Azhar University, Egypt.

${ }^{2}$ Solar and Space Research Department, NationalResearch Institute of Astronomy and Geophysics (NRIAG)

\section{ABSTRACT}

In this study, the locations of the equilibrium points of both triangular and collinear of restricted three-body problem and their stabilities are studied.This study was applied on ten binary asteroids. A code was constructed by MATHEMATICA language to obtain liberation points and their stabilities.

On the other hand, the contour of zero velocity curves was displayed for two stable and unstable binaries.

Key words: binary asteroids, liberation points, stability.

\section{INTRODUCTION}

Euler(1773)has discovered the three co-linear Lagrangian points $\left(\mathrm{L}_{1}, \mathrm{~L}_{2}\right.$ and $\left.\mathrm{L}_{3}\right)$, a few years later Lagrange discovered the remaining points $\left(\mathrm{L}_{4}\right.$ and $\mathrm{L}_{5}$ ). Sharma(1980) Studied periodic orbits of the second kind in the restricted three body problem when the more massive primary is an oblate spheroid.Sharivastavaet al.,(1983) studied equation of motion of the restricted problem of three bodies with variable mass. Gabernet al., (1991)studied a restricted four body model for the dynamic near the lagrangian points of the sun-Jupiter system.Mathlouthi(1998) Studied the infinity of periodic solution of the restricted three body problem by using a variational formulation.Llibre(1999) studied periodic and qusiperiodic orbits of the spatial three body problem. Llibreet al.,(2003) studied periodic orbits of the planer collision restricted three body problem. Llibreet al.,(2003) studied,symmetric periodic orbits of a collinear restricted three body problem. Munzoet al.,(2004) studied the families of symmetric periodic orbits in the three body problem and figure eight.Inga Jinanget al.,(2004) studied modified restricted three body problem. Reppert (2006)investigated how to refine patched conic approximation to the restricted four body problem.

In this study, the locations of the collinear and triangular points of ten binary asteroids have been computed and their stabilitieshave been determined.

2. Equation Of Motion Of TheRestricted Three-Body Problem
The restricted three-body problem refers to the dynamics of two bodies of masses $\mathrm{m} 1 \leq$ $\mathrm{m} 2$ (referred to as the primaries) that move along circles about their common center of mass, and of a third body, of infinitesimal mass, that is subject to the gravitational attraction of the primaries. The motion of the primaries is not affected by the motion of the infinitesimal mass. Fig.(2.1) illustrates the position of the third body $\mathrm{m}_{3}$ referring to the center of mass of $\mathrm{m}_{1}$ and $\mathrm{m}_{2}$, and the reference plane $(\mathrm{x}, \mathrm{y}, \mathrm{z})$.

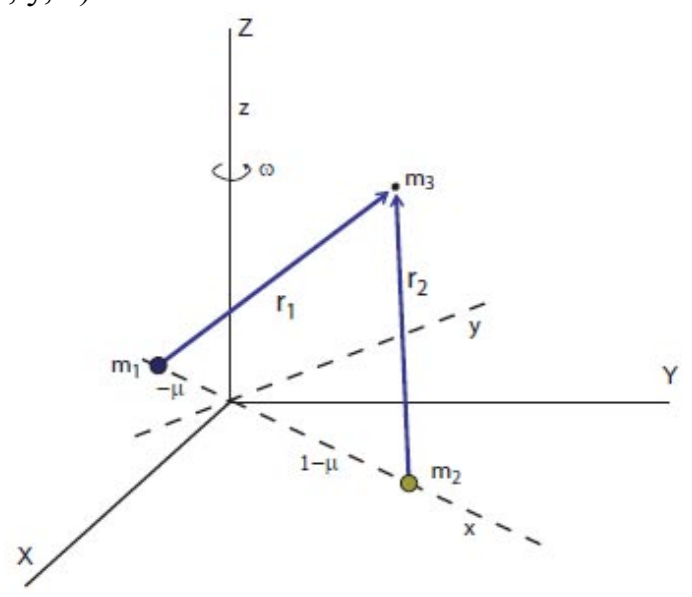

\section{Figure2.1 inertial frame of three bodies.}

The equations of motion for third body in synodic barycentric coordinate are given by (Szebehely, 1967):

$\ddot{x}-2 \dot{y}=x-\frac{\mu_{1}}{r_{1}^{3}}\left(x-x_{1}\right)-\frac{\mu_{2}}{r_{2}^{3}}\left(x-x_{2}\right)$, 
8

$\ddot{y}+2 \dot{x}=y-\left[\frac{\mu_{1}}{r_{1}^{3}}+\frac{\mu_{2}}{r_{2}^{3}}\right] y$,

$\ddot{z}=-\left[\frac{\mu_{1}}{r_{1}^{3}}+\frac{\mu_{2}}{r_{2}^{3}}\right] \quad z \quad$.

where

$\mu=\frac{m_{2}}{m_{1}+m_{2}}:$ mass ratio

$r_{1}^{2}=\left(x-x_{1}\right)^{2}+y^{2}+z^{2}$ distance from $m_{1}$ to $\mathrm{m}_{3}$,

$r_{2}^{2}=\left(x-x_{2}\right)^{2}+y^{2}+z^{2}$ :distance from $m_{2}$ to $\mathrm{m}_{3}$,

$\mu_{1}=\mathrm{Gm}_{1}=1-\mu:$ gravitational parameter for $\mathrm{m}_{1}$,

$\mu_{2}=\mathrm{Gm}_{2}=\mu \quad$ :gravitational parameter for $\mathrm{m}_{2}$,

$\mathrm{x}_{1}=-\mu_{2}=-\mu$ :distance from $\mathrm{m}_{1}$ to mass center,

$\mathrm{x}_{2}=\mu_{1}=1-\mu$ : distance from $\mathrm{m}_{2}$ to mass center.

\section{Liberation points}

At the liberation points there are zero velocity regions. So that it is very important to specify the location of these points.

Figure(3.1) showsthe liberation points $\left(\mathrm{L}_{1}\right.$, $\mathrm{L}_{2}, \mathrm{~L}_{3}, \mathrm{~L}_{4}$ and $\left.\mathrm{L}_{5}\right)$ for thetwo primary bodies $\left(\mathrm{m}_{1}\right.$ and $\mathrm{m}_{2}$ ).

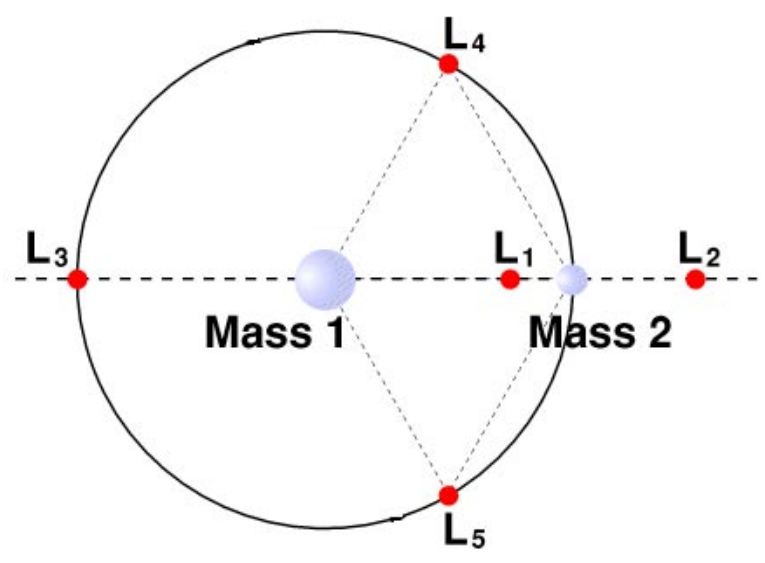

Figure3.1 : The five Lagrange Points associated with two primary bodies.

Some restrictions are considered to determine the locations of the liberation points, which are:
1- $\mathrm{m}_{3}$ lies at any point of $\left(\mathrm{L}_{1}, \mathrm{~L}_{2}, \mathrm{~L}_{3}, \mathrm{~L}_{4}\right.$, $\left.\mathrm{L}_{5}\right)$.

2- $\mathrm{m}_{3}$ is very smaller than $\mathrm{m}_{1}, \mathrm{~m}_{2}$.

3- the third mass would have zero velocity and zero acceleration where would appear permanently at rest relative to $m_{1}$ and $m_{2}$, and the equilibrium points are defined when

$\dot{\mathrm{x}}=\dot{\mathrm{y}}=\dot{\mathrm{z}}=0$,

$\ddot{x}=\ddot{y}=\ddot{z}=0$.

SubstitutingEqs. ${ }^{(3.1)}$ and $^{(3.2)}$ into

(2.1),(2.2) and (2.3) respectively, this yields

$\mathrm{x}=\frac{\mu_{1}}{\mathrm{r}_{1}^{3}}(\mathrm{x}+\mu)+\frac{\mu_{2}}{\mathrm{r}_{2}^{3}}(\mathrm{x}-(1-\mu))$,

$y=\left[\frac{\mu_{1}}{r_{1}^{3}}+\frac{\mu_{2}}{r_{2}^{3}}\right] y$,

$\left[\frac{\mu_{1}}{r_{1}^{3}}+\frac{\mu_{2}}{r_{2}^{3}}\right] \quad z=0$.

\subsection{Locationofliberationpointsof $L_{4}$ and $L_{5}$}

After some little algebraic calculations had been done to solve (3.3),(3.4)and(3.5) then it is found that

$\mathrm{x}=\frac{1}{2}-\mu$,

$y= \pm \frac{\sqrt{3}}{2}$.

So, the coordinates of $\mathrm{L}_{4}$ and $\mathrm{L}_{5}$ are being

$\mathrm{L}_{4}\left(\frac{1}{2}-\mu, \frac{\sqrt{3}}{2}\right)$ and

$\mathrm{L}_{5}\left(\frac{1}{2}-\mu,-\frac{\sqrt{3}}{2}\right)$.

3.2- Location of liberation points of $\mathrm{L}_{1}, \mathrm{~L}_{2}$ and $\mathrm{L}_{3}$

$\mathrm{L}_{1}, \mathrm{~L}_{2}$ and $\mathrm{L}_{3}$

RecallEqs. (2.1), (2.2) and (2.3) with $y=0$ as well as $\mathrm{z}=0$, then the three collinear points

$\left(L_{1}, L_{2}, L_{3}\right)$ could be found from Eq.(3.3)

Now we can calculate $\left(\mathrm{L}_{1}, \mathrm{~L}_{2}, \mathrm{~L}_{3}\right)$ from 
these eqs.

1- $\quad$ For $^{\mathrm{L}_{1}} \mathrm{~L}_{1}$ lies between masses

$m_{1}$ and $m_{2} m_{1}$ and $m_{2}$ and it can be calcul

$x-\frac{(1-\mu)}{(x+\mu)^{2}}+\frac{\mu}{(x-(1-\mu))^{2}}=0$.

2- For $\mathrm{L}_{2}$ lies outside the masse $\mathrm{m}_{2}$

and it can be calculatedfrom nonlinear equation

$x-\frac{(1-\mu)}{(x+\mu)^{2}}-\frac{\mu}{(x-(1-\mu))^{2}}=0$.

3- $\quad$ ForL $_{3}$ point lies on the negative

$\mathrm{x}$-axis and it can be calculated from nonlin

$\mathrm{x}+\frac{(1-\mu)}{(\mathrm{x}+\mu)^{2}}+\frac{\mu}{(\mathrm{x}-(1-\mu))^{2}}$

$$
=0 \text {. }
$$

\section{Jacobi Integral}

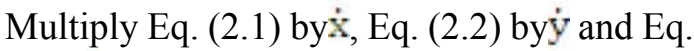
(2.3) by $\dot{z}$ to obtain

$\ddot{x} \dot{x}-2 \dot{x} \dot{y}-\dot{x} x=-\frac{\mu_{1}}{r_{1}^{3}} \dot{x}(x+\mu)-\frac{\mu_{2}}{r_{2}^{3}} \dot{x}(x-(1-\mu)$,

$\ddot{y} \dot{y}+2 \dot{x} \dot{y}-\dot{y} y=-\frac{\mu_{1}}{r_{1}^{3}} \dot{y} y-\frac{\mu_{2}}{r_{2}^{3}} \dot{y} y$,

$\ddot{z} \dot{z}=-\frac{\mu_{1}}{r_{1}^{3}} \dot{z} z-\frac{\mu_{2}}{r_{2}^{s}} z \dot{z}$.

After some algebraic calculations, the integrationwas done to obtain the zero velocity curves (Moulton, 1970)

$\frac{1}{2} v^{2}-\frac{1}{2}\left(x^{2}+y^{2}\right)-\frac{\mu_{1}}{r_{1}}-\frac{\mu_{2}}{r_{2}}=c$

where

$\frac{1}{2} \mathrm{v}^{2}$ :is kinetic energy per unit mass relative to the rotating frame,

$-\frac{\mu_{1}}{r_{1}}$ and $-\frac{\mu_{2}}{r_{2}}$ :arethe gravitational potential energy of the two primary masses respectively,

c: is called Jacobi integral, or Jacobi constant, sometimes called the integral of relative energy.

Equations (4.1) illustrates the zero velocity curves whenv $=0$.
5. Stabilityof the liberation points

\section{1 -Firstly at collinear points}

To study the motion near any of the equilibrium point $\mathrm{L}\left(\mathrm{x}_{0}, \mathrm{y}_{0}\right) . \mathrm{L}$

$$
\begin{aligned}
& x=x_{0}+\xi(5.1) y \\
& =y_{0}+\eta
\end{aligned}
$$

where $\xi$ and $\eta$ are the coordinate

and thePotentialVis

$V=\frac{1}{2}\left(x^{2}+y^{2}\right)+\frac{1-\mu}{r_{1}}+\frac{\mu}{r_{2}}$

So,Vmay be expanded by Taylor series a

$\mathrm{L}_{\mathrm{i}}(\mathrm{i}=1, \ldots .5)$ as

$\mathrm{V}=\mathrm{v}\left(\mathrm{x}_{0}, \mathrm{y}_{0}\right)+\mathrm{v}_{\mathrm{x}}\left(\mathrm{x}_{0}, \mathrm{y}_{0}\right) \xi+\mathrm{v}_{\mathrm{y}}\left(\mathrm{x}_{0}, \mathrm{y}_{0}\right) \eta+\frac{1}{2 !} \mathrm{v}_{\mathrm{xx}}\left(\mathrm{x}_{0}, \mathrm{y}_{0}\right) \xi^{2}+\mathrm{v}_{\mathrm{xy}}\left(\mathrm{x}_{0}, \mathrm{y}_{0}\right) \xi \eta$ $+\frac{1}{2 !} \mathrm{v}_{\mathrm{yy}}\left(\mathrm{x}_{0}, \mathrm{y}_{0}\right) \eta^{2}$

where

$\mathrm{V}_{x}$ is first derivative of $\mathrm{V}$ with respect to $\mathrm{x}$,

$\mathrm{V}_{\mathrm{y}}$ is first derivative of $\mathrm{V}$ with respect to $\mathrm{y}$,

$V_{x x}$ is second derivative of $V$ with respect to $x$,

$V_{y y}$ is second derivative of $V$ with respect to $y$,

The equation of motion of three body could be written in suitable form as

$\ddot{x}-2 \dot{y}=V_{x}$,

$\ddot{\mathrm{y}}-2 \dot{\mathrm{x}}=V_{\mathrm{y}}$,

$\ddot{z}=V_{z}$.

SubstitutefromEqs.(5.1) and (5.2) into Eqs. (5.4), (5.5) and (5.6) respectively then it is found that $\ddot{\xi}-2 \dot{y}=\xi V_{x x}+V_{x y} \eta$,

$\ddot{\eta}+2 \dot{\xi}=\xi V_{x y}+V_{y y} \eta$,

$\ddot{\zeta}=\zeta \mathrm{V}_{z z}$.

Let

$\xi=\alpha \mathrm{e}^{\lambda t}$,

$\eta=\beta e^{\lambda t}$.

Substitute fromEqs. (5.10) and (5.11) into Eqs (5.7), (5.8) and (5.9) respectively then it is found that,

$\left.\left(\lambda^{2}-v_{x x}\right) \alpha={ }_{(2} \lambda+v_{x y}\right) \beta$, 
M.N.Ismail, et al.

$\left.\left(2{ }^{\lambda-V_{x y}}\right) \alpha=\lambda^{2}-v_{y y}\right) \beta$.

The characteristic equation becomes (Moulton, 1970):

$\lambda^{4}+\left(4-v_{x x}-v_{y y}\right) \lambda^{2}+v_{x x} v_{y y}-v_{x y}{ }^{2}=0$.

By solvingeq. (5.14) the roots of $\lambda$ are obtained. If the roots obtained are pure (5.14) nary numbers, then $\xi$ and $\eta$ are periodic and this stable periodic solution in the vicinity of $\mathrm{x}_{0}$ and $\mathrm{y}_{0}$ can be studied as:

1- If any of the $\lambda$ roots are real or complex number, then ${ }^{\xi}$ and ${ }^{\eta}$ increase with time so that the solution is unstable. This can be happened because the solution contains constants terms in the form of exponentials.

2- If the remaining exponentials are purely imaginaries. Then the solution is stable.

$$
\begin{aligned}
& \text { To obtain the expressions } \\
& V_{x x}, V_{y y}, V_{x y} \text {, } \\
& V_{x z} \text { and } V_{y z} \text { in terms of } r_{1}, r_{2} \text { and }^{\mu_{y}} \text { let } \\
& r_{i}^{2}=\left(x-x_{i}\right)^{2}+y^{2}+z^{2}, \quad i=1,2 \text {; } \\
& A=\frac{1-\mu}{r_{1}{ }^{3}}+\frac{\mu}{r_{2}{ }^{3}} \\
& B=3\left(\frac{1-\mu}{r_{1}{ }^{5}}+\frac{\mu}{r_{2}{ }^{5}}\right) \text {, } \\
& C=3\left[\frac{1-\mu}{r_{1}^{5}}\left(x_{0}-x_{1}\right)+\frac{\mu}{r_{2}^{5}}\left(x_{0}-x_{2}\right)\right] . \\
& \mathrm{V}_{x x}=1-\mathrm{A}+3(1-\mu) \frac{\left(\mathrm{x}_{0}-\mathrm{x}_{1}\right)^{2}}{\mathrm{r}_{1}{ }^{5}}+3 \mu \frac{\left(\mathrm{x}_{0}-\mathrm{x}_{2}\right)^{2}}{\mathrm{r}_{2}{ }^{5}}, \\
& \mathrm{~V}_{\mathrm{yy}}=1-\mathrm{A}+\mathrm{By}_{0}{ }^{2} \text {, } \\
& \mathrm{V}_{z z}=-\mathrm{A}+\mathrm{Bz}_{0}{ }^{2} \text {, } \\
& \text { while } \\
& \mathrm{V}_{\mathrm{xy}}=\mathrm{C} \mathrm{y}_{0}, \mathrm{~V}_{\mathrm{xz}}=\mathrm{Cz}_{0}, \quad \mathrm{~V}_{\mathrm{yz}}=\mathrm{By}_{0} \mathrm{z}_{0} .
\end{aligned}
$$

In the case of collinear points, ${ }^{\mathrm{y}_{0}}={ }^{\mathrm{z}_{0}}=0$, so that

$\mathrm{r}_{\mathrm{i}}^{2}=\left(\mathrm{x}_{0}-\mathrm{x}_{\mathrm{i}}\right)^{2}$, i.e., $\mathrm{i}=1,2(5.18)$

$\mathrm{V}_{\mathrm{xy}}=\mathrm{V}_{\mathrm{xz}}=\mathrm{V}_{\mathrm{yz}}=0$.

Then, the equations of motion become

$\ddot{\xi}-2 \dot{y}=\xi \mathrm{V}_{x x}=\xi(1+2 \mathrm{~A})$,

$\ddot{\eta}+2 \dot{\xi}=V_{y y} \eta=\eta(1-A)$,

$\ddot{\zeta}=\zeta \mathrm{V}_{z z}=-\mathrm{A} \zeta$.

The Last equation is independent of the first twoEqs. and its solution is $\zeta=c_{1} \sin t+c_{2} \cos t$.

Therefore the motion parallel to the z-axis for small displacement is periodic with pe$\operatorname{riod} 2 \pi \pi$.Applying the values of $\mathrm{V}_{x x}, \mathrm{~V}_{x y}$ and $\mathrm{V}_{\mathrm{yy}}$ $\mathrm{V}_{\mathrm{vv}}$ in equation (5.14) yields

$\lambda^{4}+(2-A) \lambda^{2}+\left(1+A-2 A^{2}\right)=0$

Now there are three values of A corresponding to the three Lagrangian points $L_{1}$, $\mathrm{L}_{2}, \mathrm{~L}_{3}$ obtained from equations (3.2.1), (3.2.2) and (3.2.3) respectively. It can be shown that the values of $L_{1}, L_{2}$ and $L_{3}$ the next condition is verified.

$1+\mathrm{A}-2 \mathrm{~A}^{2}<0$

While values of $\mu$ up to its limit $1 / 2$.Then, the four roots of equation (5.22) consist of two real roots, numerically equal but opposite in sign, and two conjugate pure imaginary roots. Then the solution for the straight-line case is unstable and the orbit becomes spiral.

\section{2-Secondly triangular points:}

The coordinate of the triangular equilibrium points $\mathrm{L}_{4}, \mathrm{~L}_{5}$ are

$\mathrm{x}_{0}=\frac{1}{2}-\mu \quad, \mathrm{y}_{0}= \pm \frac{\sqrt{3}}{2}$

$\mathrm{AtL}_{4}$ from Eqs (5.15),(5.16),(5.17) and(5.22) it is found that

$\mathrm{V}_{\mathrm{xx}}=3 / 4, \mathrm{~V}_{\mathrm{yy}}=9 / 4, \mathrm{~V}_{z z}=-1$,

$V_{v u}=\frac{3 \sqrt{3}}{4}(1-2 \mu), \quad V_{x z}=$
$V_{y z} V_{y z}=0$.

The equations of motion at ${ }^{\mathrm{L}_{4}}$ become 


\section{STUDYING STABILITY OF THE LIBERATION POINTS OFBINARY ASTEROIDES}

$\ddot{\xi}-2 \dot{\eta}=3 / 4 \xi+\frac{3 \sqrt{3}}{4}(1-2 \mu) \xi$,

$\ddot{\eta}+2 \dot{\xi}=\frac{3 \sqrt{3}}{4}(1-2 \mu) \xi+\frac{9}{4}$,

$\ddot{\zeta}=\zeta \mathrm{V}_{z z}=-\zeta$.

The Last equation is independent of the first two and its solution is

$\zeta=c_{1} \sin t+c_{2} \cos t$.

so that the motion parallel to the z-axis for small displacement and the solution is periodic withperiod $2^{\pi}$.

As the same way to determine the characteristic equation for collinear points it is found that thecharacteristic equation for $\mathrm{L}_{4}$ becomes

$$
\lambda^{4}+\lambda^{2}+\frac{27}{4} \mu(1-2 \mu)=0 \text {. }
$$

If $\mu \leq_{1 / 2}$ and if $1-27 \mu(1-2 \mu) \geq 0$ the roots are pure imaginary.

The inequality may be written as $1-27 \mu(1-2 \mu)=\varepsilon$,

where ${ }^{\varepsilon}$ is a positive quantity whose limit is zero.

The solution of this equation is

$\mu=\frac{1}{2} \pm \sqrt{\frac{23+4 \varepsilon}{108}}$.

Since ${ }^{\mu}$ represents the mass ratio, which is less than $1 / 2$ the negative sign must be tak

en at the limit, ${ }^{\varepsilon=0} \rightarrow \mu=0.0385 .0$

Therefore if ${ }^{\mu<0.0385}$ the roots become pure imaginaries and the motion of the particle displaced from the equilibrium point is oscillatory in form, hence the particle will remain in the vicinity ofequilibrium point and the motion become stable.

If $^{\mu>0.0385}$ the roots become complex and the orbits become spiral.

The spiral orbits asymptotically approach the triangular libration points or depart from them;therefore, the motionbecomes unstable.

\section{RESULTS AND CONCLUSION}

A code was constructed by MATHEMATICA language, and applied on the ten binary asteroids to obtain liberation points, mass ratio and drawing contour plot of zero velocity curves. Selected two binary asteroids One of them its triangular points stable and another unstable at $\mathrm{L}_{4}$ and $\mathrm{L}_{5}$. By usingthese relations to determine mass ratio from half- diameter ofeach mass binary asteroids as

$$
\begin{aligned}
& \mu=\frac{m_{2}}{m_{1}+m_{2}} \\
& =\frac{\frac{4}{3} \pi \rho R^{3}{ }_{2}}{\frac{4}{3} \pi \rho\left(R^{3}{ }_{1}+R^{3}{ }_{2}\right)} \\
& =\frac{R^{3}{ }_{2}}{R^{3}{ }_{1}+R^{3}{ }_{2}} .
\end{aligned}
$$

Tables (6.1) and (6.2) show the results for the given data of two asteroid diameters(D1 and D2)and the distance between them $\left(\mathrm{R}_{\mathrm{b}}\right)$, which are usedby the code to determine the liberation points $\left(\mathrm{L}_{1}, \mathrm{~L}_{2}, \mathrm{~L}_{3}, \mathrm{~L}_{4}\right.$ and $\left.\mathrm{L}_{5}\right)$ for the ten binary asteroids. Figures (6.1) and (6.2)display the contours of zern velnrity rurves for stable binary 1996FG3 at t $^{\mu}=0.02829$ and for unstablebinary 1999 DJ4 at $\mu=0.1111$ respectively. 
M.N.Ismail, et al.

Table 6.1: collinear points and stability for binary system.

http://echo.jpl.nasa.gov/ lance/binary.neas.html.

\begin{tabular}{|c|c|c|c|c|c|c|c|c|c|}
\hline No & Binary & $\begin{array}{c}D_{1} \\
(\mathrm{~km})\end{array}$ & $\begin{array}{c}D_{2} \\
(\mathrm{~km})\end{array}$ & $\begin{array}{c}R_{b} \\
(\mathrm{~km})\end{array}$ & $\mu$ & $\begin{array}{c}L_{1} \\
(\mathrm{~km})\end{array}$ & $\begin{array}{c}L_{2} \\
(\mathrm{~km})\end{array}$ & $\begin{array}{c}L_{3} \\
(\mathrm{~km})\end{array}$ & $\begin{array}{c}\text { Stability } \\
\text { For } \\
L_{1}, L_{2}, L_{3}\end{array}$ \\
\hline 1 & VH 1991 & 1.1 & 44. & 3.2 & 0601. & 2.212 & 3.959 & -3.28 & unstable \\
\hline 2 & AW1 1994 & 1 & 49. & 2.3 & 10526. & 1.337 & 2.901 & -2.40 & unstable \\
\hline 3 & FG3 1996 & 1.5 & 465. & 2.6 & 02892. & 2.009 & 3.118 & -2.63 & unstable \\
\hline 4 & PG 1998 & 9. & 27. & 1.5 & 02629. & 1.127 & 1.79 & -1.51 & unstable \\
\hline 5 & DJ4 1999 & 35. & 175. & 8. & 11111. & 47. & 9093. & $836 .-$ & unstable \\
\hline 6 & KW4 1999 & 1.5 & 57. & 2.54 & 05201. & 1.804 & 3.124 & -5.595 & unstable \\
\hline 7 & DP107 2000 & 8. & 328. & 2.6 & 06447. & 1.77 & 2.955 & -2.699 & unstable \\
\hline 8 & UG11 2000 & 26. & 156. & 4. & 17763. & 189. & 508. & $429 .-$ & unstable \\
\hline 9 & SL9 2001 & 8. & 224. & 1.4 & 02148. & 1.117 & 1.657 & -1.412 & unstable \\
\hline 10 & CE26 2002 & 3 & 21. & 5.1 & 00034. & 4.854 & 5.349 & -5.1007 & unstable \\
\hline
\end{tabular}

Table 6.2: triangular points and stabilities for binary system.

\begin{tabular}{|c|c|c|c|c|c|c|}
\hline \multirow{2}{*}{$\begin{array}{c}\text { No } \\
1\end{array}$} & \multirow{2}{*}{$\begin{array}{c}\text { Binary } \\
\text { VH } 1991\end{array}$} & \multicolumn{2}{|c|}{$\begin{array}{c}L_{4} \\
(\mathrm{x}, \mathrm{y})\end{array}$} & \multicolumn{2}{|c|}{$\begin{array}{c}L_{5} \\
(\mathrm{x}, \mathrm{y})\end{array}$} & \multirow{2}{*}{$\begin{array}{c}\text { Stability } \\
L_{4}, L_{5} \text { For } \\
\text { Unstable }\end{array}$} \\
\hline & & 1.407 & 2.771 & 1.407 & -2.771 & \\
\hline 2 & AW1 1994 & 0.908 & 1.991 & 0.908 & -1.991 & Unstable \\
\hline 3 & FG3 1996 & 1.285 & 2.251 & 1.285 & -2.251 & Stable \\
\hline 4 & PG 1998 & 0.711 & 1.299 & 0.711 & -1.299 & Stable \\
\hline 5 & DJ4 1999 & 0.311 & 0.692 & 0.311 & -0.692 & unstable \\
\hline 6 & KW4 1999 & 1.139 & 2.199 & 1.139 & -2.199 & unstable \\
\hline 7 & DP107 2000 & 1.132 & 2.251 & 1.132 & -2.251 & unstable \\
\hline 8 & UG11 2000 & 0.129 & 0.346 & 0.129 & -0.346 & unstable \\
\hline 9 & SL9 2001 & 0.670 & 1.212 & 0.670 & -1.212 & stable \\
\hline 10 & CE26 2002 & 2.584 & 4.42 & 2.584 & -4.42 & stable \\
\hline
\end{tabular}


STUDYING STABILITY OF THE LIBERATION POINTS OFBINARY ASTEROIDES

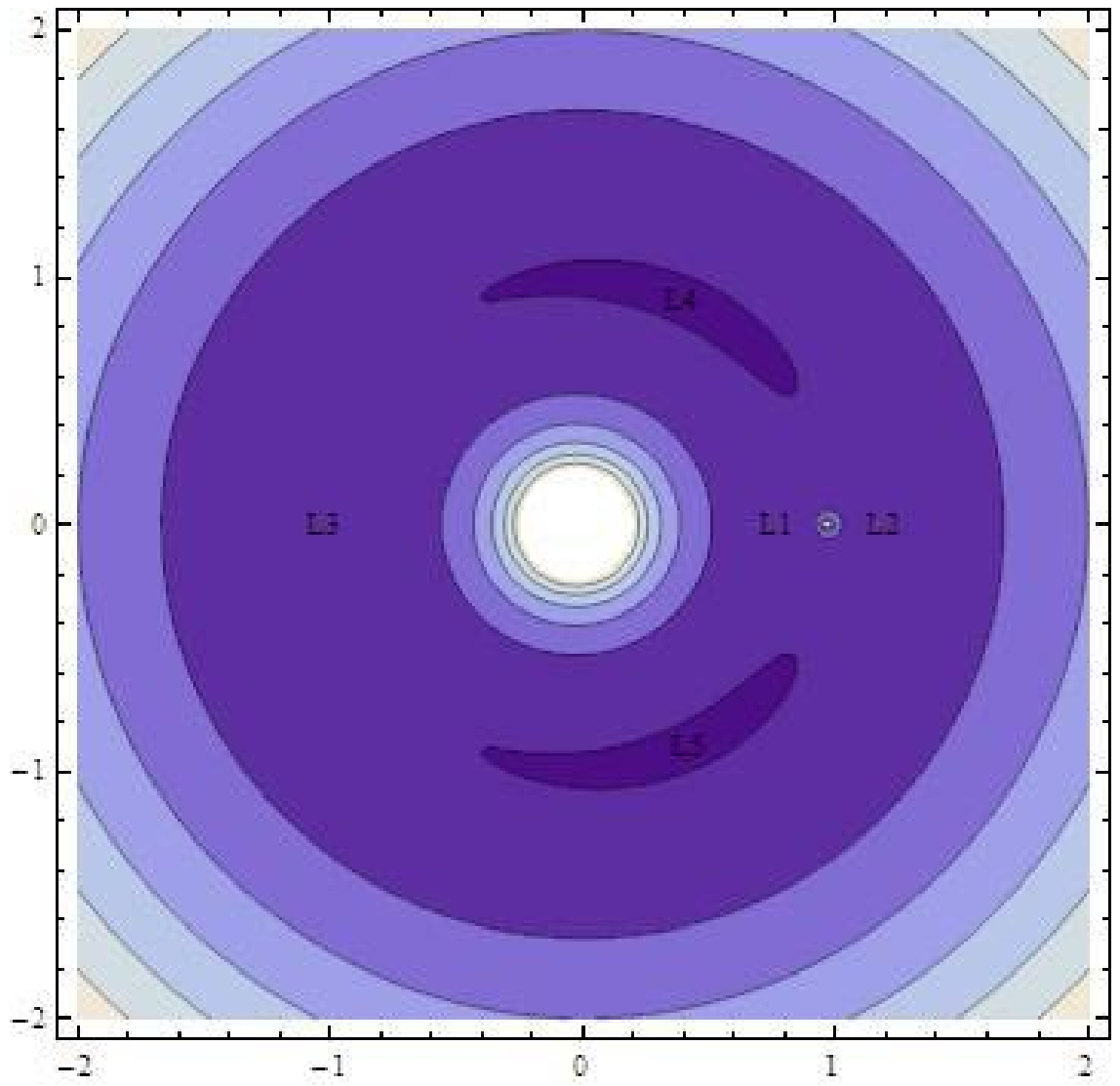

Figure 6.1: Contour plotfor 1996 FG3 binary system with $\mu=0.02829$

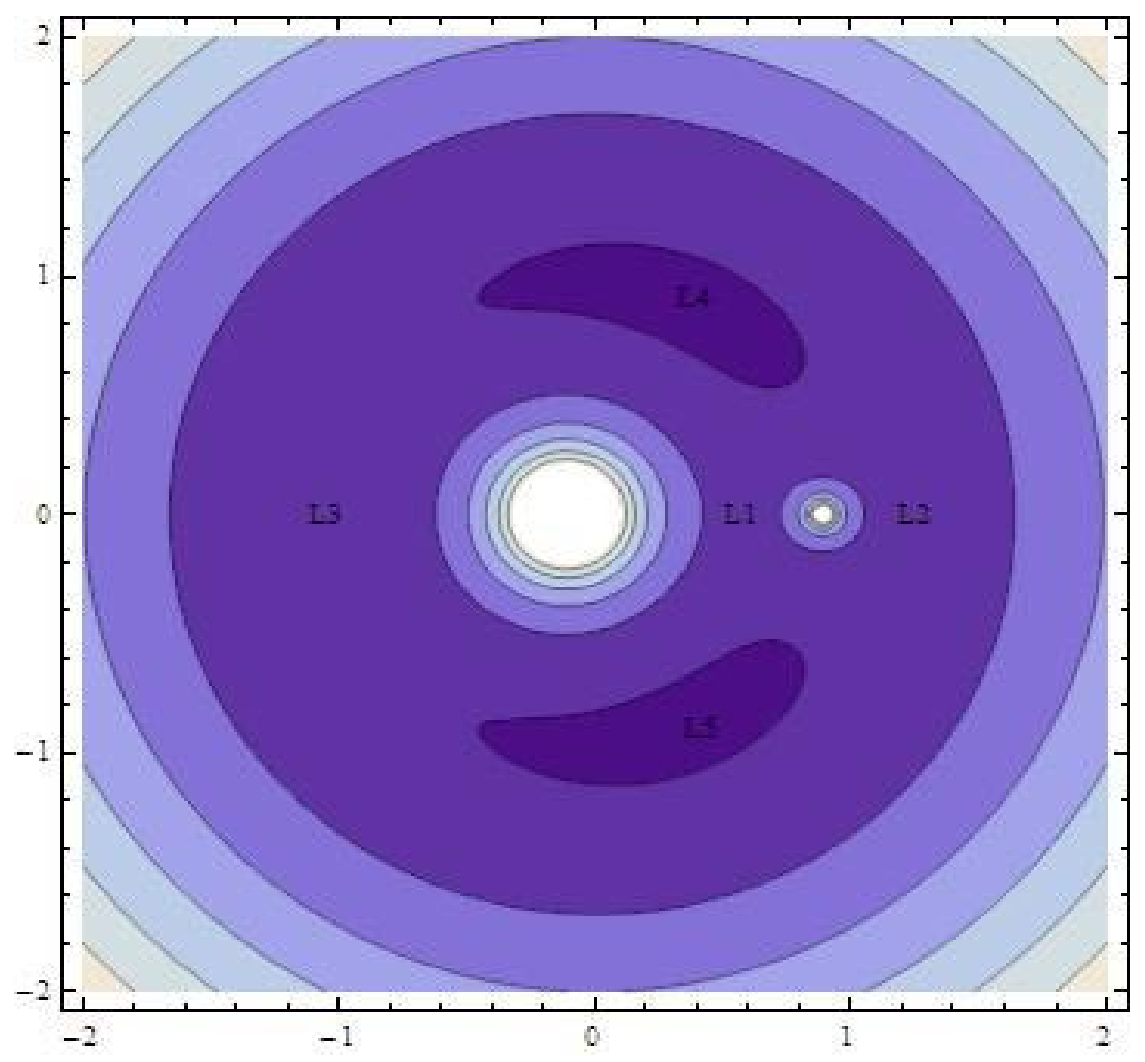

Figure 6.2: Contour plotfor1999 DJ4 binary systemwith $\mu=0.1111$ 
M.N.Ismail, et al.

REFERENCES:

Benner, L. http://echo.jpl.nasa.gov/ lance/binary.neas. html.

Gabern, F. andJorba, A; 1991. "Restricted four and five body problem in the solar system" universitata de Barcelona gran via 585,08007 Barcelona, Spain.

Inga Jinang and Lia-chin Yeh.;2004."The modified restricted three body problem". The environment of evolution of douple and multiple stars .IA,UNAM.

Euler,Leonhard, 1773, De moturectilineotriumcorporum se mutuoattrahentium (http:/ / www. math. dartmouth. edu/ euler/ docs/ originals/E327. pdf)

Llibre, J.;1999."Periodic and qusi-periodic orbits of the spatial three body problem".MontseCobera.

Llibre, J. and Montserrat, C.; 2003. "Periodic orbits of a collinear restricted three body problem" Celestial mechanics and dynamical astronomy $86: 163-183$, Netherlands.

Mathlouthi, S.;(1998).'Periodic orbits of the restricted three body problem". American mathematical society, Volume 850 , No 6 .
Moulton, F. R.; 1970.”An Introduction to Celestial Mechanics". Dover, New York.

Munzo, F.J, Galant. J. and Freire, J.;2004.”The families of symmetric periodic orbits in the three body problem and figure eight" Monografias de la Real Acadmia de ciencias de zaragoz.25: 229-240.

Reppert, T.R.; 2006. "the patched conic approximation to the restricted four -body problem". Monografias de la Real Acadmia de ciencias de zaragoz.30: 133-146.

Sharma,R. K.; 1980."Periodic orbits of the second kind in the restricted three body problem when the more massive primary “. VirkarmSarabahaiSpace Center, Trivandrum, India.

Sharivastava. A.K. andIshwar B..;1983. "Equation of motion of the restricted problem of three bodies with variable mass". Department of mathematics .india college engineering, Bihar, India

Szebehely. V.G.; 1967. "Theory of orbits: The restricted problem of Three-Bodies". Academic Press Inc, New York. 